\title{
Perfil epidemiológico dos casos de Leishmaniose Tegumentar Americana no estado do Maranhão no período de 2015 a 2017
}

\author{
Epidemiological profile of American Cutaneous Leishmaniasis cases in the state of \\ Maranhão from 2015 to 2017
}

Benjamin Franklin Pinheiro Alencar ${ }^{1 *}$, Ivan Abreu Figueiredo ${ }^{1}$

\begin{abstract}
Resumo: A Leishmaniose Tegumentar Americana (LTA) é uma doença causada por protozoários do gênero Leishmania, transmitida ao homem pela picada de mosquitos flebotomíneos. As formas clínicas da leishmaniose tegumentar americana são as mais variadas. Na leishmaniose cutânea (LC), na sua fase aguda, geralmente o quadro se inicia com pápula nos locais da picada do inseto que, basicamente, acometem áreas descobertas do corpo. Na leishmaniose mucosa (LM), a lesão atinge de preferência as vias aéreas superiores. O Brasil é considerado o terceiro país com o maior número de casos novos em todo o mundo, com uma média de 21.000 casos anuais. Trata-se de uma doença endêmica no Brasil, tendo distribuição em todo território nacional, com maior casuística na Pré-Amazônia Maranhense. O estado do Maranhão é o segundo em número absoluto de casos de LTA dentre todos os estados do país. OBJETIVOS: O objetivo principal desse estudo é apresentar o perfil epidemiológico dos casos de Leishmaniose Tegumentar Americana no Estado do Maranhão, no período de 2015 a 2017, a partir de dados coletados no SINAN de forma a contribuir para a melhoria das informações disponibilizadas da doença no estado. METODOLOGIA: Este trabalho consiste em uma pesquisa observacional, descritiva, quantitativa com base nos dados notificados e cadastrados no Sistema de Informações de Agravos de Notificações. Os mesmos serão transcritos para o programa Microsoft Excel e tabulados para posterior realização da análise estatística descritiva através do cálculo das frequências relativas das respostas dadas, sendo os resultados apresentados em porcentagens na forma de gráficos e tabelas.
\end{abstract}

Palavras chaves: Leishmania. Perfil epidemiológico. Zoonose.

\begin{abstract}
American Cutaneous Leishmaniasis (LTA) is a disease caused by protozoa of the genus Leishmania, transmitted to humans by the bite of sandfly mosquitoes. The clinical forms of American cutaneous leishmaniasis are the most varied. In cutaneous leishmaniasis (LC), in its acute phase, the picture usually starts with a papule at the insect bite sites, which basically affect areas of the body. In mucosal leishmaniasis (LM), the lesion preferably reaches the upper airways. Brazil is considered the third country with the highest number of new cases in the world, with an average of 21,000 cases per year. It is an endemic disease in Brazil, with distribution throughout the national territory, with a greater number of cases in the preAmazônia Maranhense. The state of Maranhão is the second in absolute number of cases of LTA among all the states of the country. OBJECTIVES: The main objective of this project is to present the epidemiological profile of cases of American Tegumentary Leishmaniasis in the State of Maranhão, from 2015 to 2017, based on data collected in SINAN in order to contribute to the improvement of the information available on the disease. state. METHODOLOGY: This work consists of an observational, descriptive, quantitative research based on the data reported and registered in the Notification of Injury Information System. They will be transcribed to the Microsoft Excel program and tabulated for subsequent statistical and descriptive analysis by calculating the relative frequencies of the given answers, the results being presented in percentages in the form of graphs.
\end{abstract}

Key words: Leishmania. Epidemiological profile. Zoonosis.

${ }^{1}$ Universidade CEUMA, Medicina (Maranhão, Brasil)

*Autor correspondente: Ivan Abreu Figueiredo, Universidade CEUMA, Campus Renascença. Endereço: Rua Josué Montello, n¹, Renascença II - CEP 65.075-120 - São Luís/MA. Email: ivanfig@terra.com.br 


\section{Introdução}

A Leishmaniose Tegumentar Americana (LTA) é uma doença causada por protozoários do gênero Leishmania, transmitida ao homem pela picada de mosquitos flebotomíneos (Ordem Díptera; Família Psychodidae; Subfamília Phlebotominae). No Brasil, existem atualmente seis espécies de Leishmania responsáveis pela doença e mais de 200 espécies de flebotomíneos relacionados com sua transmissão. Trata-se de uma doença que acompanha o homem desde tempos remotos e que tem apresentado, nos últimos 20 anos, um aumento do número de casos e ampliação de sua ocorrência geográfica, sendo encontrada atualmente em todos os Estados brasileiros, sob diferentes perfis epidemiológicos ${ }^{1,2,3}$.

A leishmaniose tegumentar constitui um problema de saúde pública em 85 países, distribuídos em quatro continentes (Américas, Europa, África e Ásia), com registro anual de 0,7 a 1,3 milhão de casos novos. É considerada pela Organização Mundial da Saúde ${ }^{4}$ como uma das seis mais importantes doenças infecciosas no mundo ${ }^{3}$.

O Brasil é considerado o terceiro país com o maior número de casos novos em todo o mundo, com uma média de 21.000 casos anuais, aproximadamente, no período de 2009 a 2013, representando $10 \%$ de todos os casos registrados no mundo, perdendo apenas para Síria e Afeganistão ${ }^{4}$. A doença atinge todas as regiões brasileiras e em 2014 observaram-se 20.418 casos novos ${ }^{5}$. A LTA é uma das afecções dermatológicas que merece mais atenção pelos serviços de saúde públicos, devido à sua magnitude, como também pelo fato de se relacionar ao risco de ocorrência de deformidades que pode produzir nos doentes afetados, além do envolvimento psicológico que se reflete nos campos social e econômico, pois, na maioria dos casos, a parasitose é considerada uma doença ocupacional ${ }^{3}$.

A principal forma de transmissão da doença é pela picada de inseto, os flebotomíneos (vetor), que no Brasil são do subgênero Lutzomia, que ao picar hospedeiros parasitados como cães, equinos, marsupiais, roedores, raposas, transmitem o parasito ao homem, caracterizando o ciclo antropozoonótico ${ }^{6}$, ${ }^{7}$ da doença.

Entre as espécies de Leishmania spp. consideradas patogênicas ao homem, as que causam a doença tegumentar no país com maior prevalência são $L$. braziliensis $L$. guyanesis e L. amazonensis $6,8,9$.

As formas clínicas da leishmaniose tegumentar americana são as mais variadas. $\mathrm{Na}$ fase aguda da leishmaniose cutânea, geralmente o quadro se inicia com pápula nos locais da picada do inseto que, basicamente, acometem áreas descobertas do corpo. Em uma ou duas semanas as lesões podem se resolver ou se tornar nódulos, ulcerar e, eventualmente, cicatrizar em um período de seis meses a três anos. A úlcera pode ser única, frequentemente com bordas elevadas, fundo granuloso, é geralmente indolor e pode atingir de alguns milímetros a vários centímetros de diâmetro ${ }^{10,11}$. $\mathrm{Na}$ leishmaniose mucosa, a lesão atinge de preferência as vias aéreas superiores. Estima-se que indivíduos que tiveram acometimento de pele prévio têm risco aumentado para a doença, de forma que muitas vezes não se detecta esta lesão, suspeitando-se de uma infecção subclínica. Posteriormente, evolui para lesão ulcerada com comprometimento da mucosa, nasofaringe, cavidade oral e laringe. Caracteriza-se por comprometimento ora ulcerativo e multilante ora com aumento de volume das partes moles e hiperemia, mas sem destruição importante. As queixas mais comuns no acometimento nasal são: obstrução, epistaxes, rinorréia e crostas; da faringe, odinofagia; da laringe, 
rouquidão e tosse; da cavidade oral, feridas na boca 6 .

Outra forma cutânea de acometimento é a leishmaniose cutâneo anérgica difusa que apresenta um comprometimento dérmico extenso e anérgica ao teste intradérmico de Montenegro. Caracteriza-se por nódulos, máculas, pápulas e placas infiltradas em todo corpo que geralmente não ulceram. Tem uma baixa resposta ao tratamento e sua recidiva é frequente ${ }^{12,13}$. Esta forma de LTA é frequentemente encontrada na região Norte e no estado do Maranhão. As lesões apresentam limites imprecisos, que se confundem com a pele normal, lembrando a hanseníase virchowiana ${ }^{14}$.

A LTA é uma doença de notificação compulsória, ou seja, todo caso efetivamente confirmado deve ser notificado por intermédio da ficha de investigação, padronizada pelo Ministério da Saúde, ao Sistema de Informações de Agravos de Notificação (SINAN) pelos serviços de saúde públicos, privados ou filantrópicos ${ }^{2,15}$.

Logo, este estudo teve por objetivo traçar o perfil epidemiológico dos casos de Leishmaniose Tegumentar Americana no Estado do Maranhão, no período de 2015 a 2017, a partir de dados coletados no, Sistema de Informação de Agravos de Notificação (SINAN) do Ministério da Saúde, de forma a contribuir para a melhoria das informações disponibilizadas sobre a doença no Estado.

\section{Metodologia}

\section{Tipo de estudo}

Trata-se de um estudo observacional descritivo quantitativo.

\section{Local}

Dados disponíveis no Sistema Nacional de Notificações e Agravos (SINAN), do Ministério da Saúde, disponibilizados para consulta pública pelo portal eletrônico saude.gov.br. Os dados coletados são referentes ao estado do Maranhão localizado no nordeste brasileiro que possui área total de $331.936 .949 \mathrm{~km}^{2}$ e uma população de 6.574.789 pessoas. Com 217 municípios, tem sua população distribuída de forma heterogênea em seu território, com densidade demográfica de $19,81 \mathrm{hab} / \mathrm{Km}^{2}$ (IBGE, 2010), além de representar uma área de confluência entre os biomas Cerrado, Amazônia e Caatinga, tendo formações características como: Mata de Cocais e Baixada, resultando em vários ecossistemas diferentes ${ }^{15,16}$.

\section{Período de coleta de dados}

Os dados foram coletados de maio de 2018 a julho de 2018.

\section{Amostra}

As variáveis escolhidas para a análise foram categorizadas em dois grupos: variáveis epidemiológicas e variáveis clínicas. As principais variáveis obtidas para as informações epidemiológicas são: número de casos, sexo, escolaridade, faixa etária raça e zona de residência. Enquanto as principais variáveis obtidas para as informações clínicas são: tipo de entrada, critério de confirmação, forma clínica, evolução do caso e número de óbitos. As informações relacionadas ao estudo serão obtidas mediante dados fornecidos pela Secretaria Estadual da Saúde do Estado do Maranhão, contidos no Banco de Dados do Sistema de Informação de Agravos de Notificação (SINAN), no qual constam dados dos casos de LTA. Os registros do Banco de Dados do SINAN foram aqueles em que a notificação da LTA, no Maranhão, estivesse devidamente compreendida no período de 2015 a 2017. 


\section{Análise estatística}

Após a realização da coleta de dados, os mesmos foram transcritos para o programa Microsoft Excel e tabulados para posterior realização da análise estatística descritiva através do cálculo das frequências relativas das respostas dadas, sendo os resultados apresentados em porcentagens na forma de gráficos, com o auxílio do software Microsotf Office Excel 2016 (Versão Windows 10). Os dados de 2015, 2016 e 2017 corresponderam aos casos validados pela Gerência Técnica de Leishmanioses/CGDT/DEVIP/SVS/MS.

\section{Aspectos éticos}

Por se tratar de uma análise fundamentada em banco de dados secundários e de domínio público, o estudo não foi encaminhado para apreciação de um Comitê de Ética em Pesquisa, mas ressalta-se que foram tomados os cuidados éticos que preceituam a Resolução 466/12, do Conselho Nacional de Saúde ${ }^{17}$.

\section{Resultados e discussão}

No período de 2015 a 2017 foram registrados 3.946 casos confirmados de LTA, de acordo com os dados obtidos pelo SINAN, em residentes do estado do Maranhão, com uma média de 1.315 casos por ano. No Gráfico 1 é possível observar que os maiores percentuais de casos foram registrados em 2015 $(n=1797,45,6 \%)$ e o menor percentual registrado foi em 2016 ( $n=1.071,27,1 \%)$.

A Tabela 1 apresenta as variáveis demográficas relativas aos casos da respectiva doença. A baixa escolaridade foi um fator relevante, considerando que em $58,3 \%$ dos casos, os indivíduos tinham baixa escolaridade: eram analfabetos ou com referência ao ensino fundamental. A faixa etária mais acometida foi a de 20 a 59 anos (63,6\%). Demonstrou-se que a LTA se apresentou com maior frequência em indivíduos de cor parda $(68,9 \%)$ e em residentes da zona rural $(51,5 \%)$. Quanto a variável sexo, os indivíduos do sexo masculino foram os mais acometidos (71,3\%).

Na Tabela 2, pode ser observada a descrição das variáveis clínicas dos casos confirmados de LTA em residentes do estado do Maranhão. No período estudado, $93,5 \%$ dos casos de LTA foi caracterizada como casos novos, enquanto que $5,2 \%$ caracterizavam-se como recidivas. A forma clínica mais prevalente foi a cutânea, sendo representada por $95,6 \%$ dos casos. Ocorreram apenas 03 óbitos que tiveram a LTA como causa básica, o percentual de cura foi de $53,3 \%$.

No período analisado foram notificados 3.946 casos, com a média anual de 1.315 casos, o que classifica o Maranhão como uma importante área endêmica da LTA no nordeste do Brasil. Dos quais a maior prevalência ocorreu em indivíduos do sexo masculino, mesmo fato observado em outros estudos 18, 19. Fatores hormonais ligados à exposição ao vetor têm sido responsabilizados pelo aumento do risco deste gênero citado 20 .

Em relação à escolaridade, notou-se a predominância de baixa escolaridade, uma vez que a grande maioria dos indivíduos se caracterizava como analfabetos ou com o ensino fundamental incompleto. $\mathrm{O}$ elevado índice de indivíduos com baixo nível de escolaridade acometido pela LTA sugere que esta seja uma doença que acomete preferencialmente pessoas de baixo nível socioeconômico ${ }^{21}$.

A maior prevalência da LTA ocorreu em adultos, com idade entre 20 a 59 anos, com a ocorrência de 3 casos de óbito no Estado. Esse dado se relaciona à realização de atividades laborais e de 


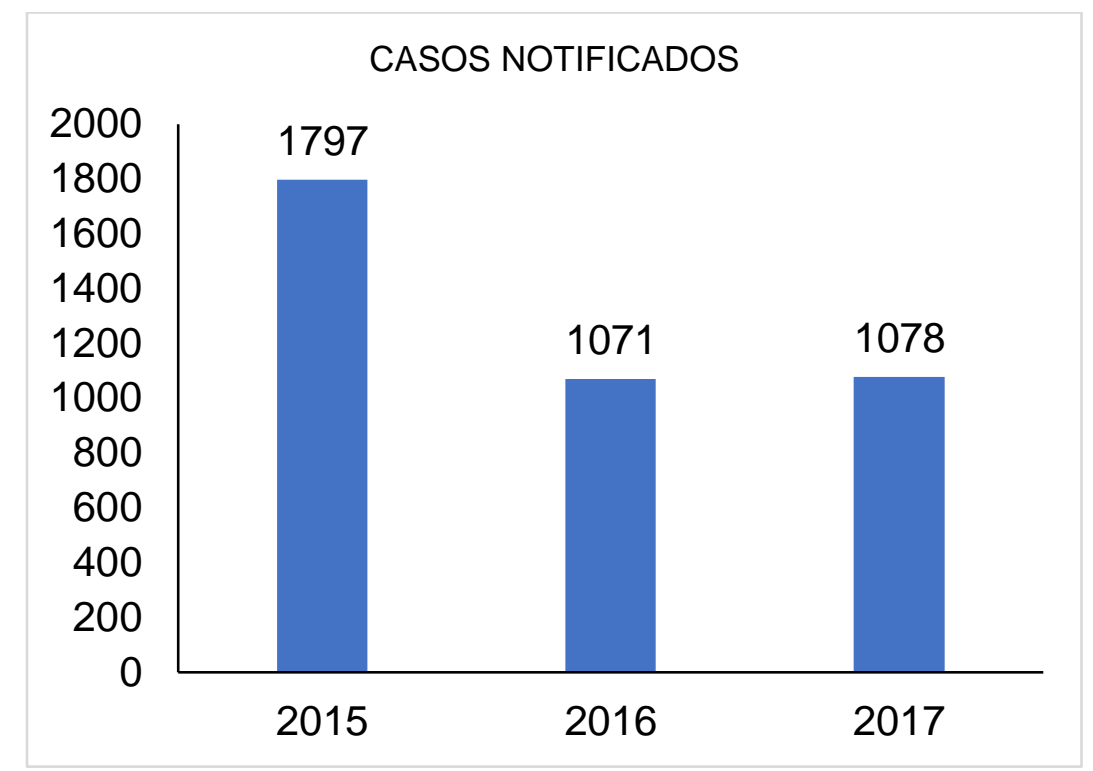

Figura 1: Números de casos de Leishmaniose Tegumentar Americana notificados no estado do Maranhão, no período 2015-2017. Fonte: Ministério da Saúde/SVS - Sistema de Informação de Agravos de Notificação - SINAN $\mathrm{NET}^{9}$.

lazer executadas por indivíduos adultos que podem aumentar as chances de exposição ao vetor e aquisição da doença 22.

Esse estudo constata que os indivíduos de raça parda são os mais acometidos pela LTA em decorrência de a região Nordeste apresentar, em sua maioria, uma população parda, com $64,5 \%$, seguida da cor branca, com $29,7 \%$ 23.

A maioria dos casos de LTA ocorreu na zona rural. As características ambientais dessas habitações favorecem a infecção, pois é comum no domicílio e seus anexos, as condições ideais para a atração e presença do flebótomo ${ }^{24}$.

A confirmação dos casos baseada em parâmetros clínico-epidemiológicos ocorreu para $1.145(29 \%)$ casos de LTA. Em $2.801 \quad(71 \%)$ dos indivíduos foi realizado diagnóstico laboratorial. Embora - diagnóstico laboratorial tenha correspondido ao critério mais utilizado para presença de LTA, nesse estudo houve um percentual de não realização destes testes para o diagnóstico reportado da LTA. Entretanto, uma análise definitiva requer a demonstração do parasita através de métodos parasitológicos ${ }^{25}$. Desta forma, o diagnóstico de certeza da LTA é feito pelo encontro do parasito, ou de seus produtos, nos tecidos ou fluidos biológicos dos hospedeiros ${ }^{26}$. Portanto, recomenda-se a confirmação do diagnóstico por método parasitológico antes do início do tratamento, principalmente naqueles casos com evolução clínica e lesões atípicas fora do habitual e/ou má resposta a tratamento anterior ${ }^{5}$.

As características clínicas relacionadas com os dados analisados demonstraram uma proporção de recidivas apresentadas no estudo de $5,2 \%$. As principais causas de recidiva da LTA são a utilização de doses menores do que a quantidade recomendada ocasionando um tratamento inadequado e também a toxicidade destes medicamentos o que leva os pacientes a desistirem do tratamento antes de seu término ${ }^{27}$. 
Tabela 1. Variáveis demográficas dos casos de Leishmaniose Visceral confirmados em residentes do estado do Maranhão nos anos de 2015 a 2017. Fonte: Ministério da Saúde/SVS - Sistema de Informação de Agravos de Notificação SINAN NET (2018).

\begin{tabular}{|c|c|c|}
\hline VARIÁVEL & NÚMERO DE CASO ( $\mathrm{n}=3946)$ & $\%$ \\
\hline Sexo & & \\
\hline Masculino & 2810 & 71,3 \\
\hline Feminino & 1136 & 28,7 \\
\hline \multicolumn{3}{|l|}{ Escolaridade } \\
\hline Analfabeto & 385 & 9,7 \\
\hline Ensino Fundamental Incompleto & 1918 & 48,6 \\
\hline Ensino Fundamental Completo & 232 & 5,9 \\
\hline Ensino Médio Incompleto & 225 & 5,7 \\
\hline Ensino Médio Completo & 290 & 7,4 \\
\hline Ensino Superior Incompleto & 16 & 0,4 \\
\hline Ensino Superior Completo & 41 & 1 \\
\hline Sem Informação & 839 & 21,3 \\
\hline \multicolumn{3}{|l|}{ Faixa etária } \\
\hline$<10$ anos & 277 & 7 \\
\hline 10 a 19 anos & 596 & 15,1 \\
\hline 20 a 59 anos & 2510 & 63,6 \\
\hline$>60$ anos & 563 & 14,3 \\
\hline \multicolumn{3}{|l|}{ Raça } \\
\hline Branca & 575 & 14,6 \\
\hline Preta & 416 & 10,6 \\
\hline Amarela & 70 & 1,8 \\
\hline Parda & 2719 & 68,9 \\
\hline Indígena & 77 & 1,9 \\
\hline \multirow{2}{*}{\multicolumn{3}{|c|}{ Zona de residência }} \\
\hline & & \\
\hline Urbana & 1777 & 45 \\
\hline Rural & 2031 & 51,5 \\
\hline Periurbana & 25 & 0,6 \\
\hline Sem Informação & 113 & 2,9 \\
\hline
\end{tabular}

A forma clínica que mais acometeu a população maranhense foi a cutânea. Essa forma apresenta-se sempre mais prevalente, devido ao fato de a forma mucosa costumar ocorrer como evolução da forma cutânea, principalmente por conta do tratamento inadequado, demora ou não realização do mesmo ${ }^{28}$.

Quanto à evolução da doença foi observada uma grande porcentagem de evolução dos pacientes para a cura e baixa porcentagem de evolução para óbito causado pela LTA, o que caracteriza que - protocolo de tratamento e acompanhamento está sendo satisfatório para os casos de LTA.

\section{Conclusão}

Este estudo contribuiu para o conhecimento do perfil epidemiológico e 
clínico da Leishmaniose Tegumentar Americana, no estado do Maranhão, compreendido entre os anos de 2015 a 2017, utilizando dados compilados do SINAN/Ministério da Saúde, contribuindo para um melhor conhecimento da doença.

No período analisado foram notificados 3.946 casos. Os dados demográficos demonstraram que a maioria dos casos ocorreram entre indivíduos do sexo masculino com baixa escolaridade, na faixa etária de 20 a 59 anos, da raça parda que residem na zona rural. Por outro lado, as variáveis clínicas expuseram que a maioria dos casos foi tida como novos, por meio do critério de confirmação clínico-laboratorial, com maior constatação da forma cutânea, evoluindo para a cura, apresentando baixa taxa de letalidade no período da pesquisa.

No entanto, é necessária a mobilização constante de recursos para que os planos e ações de controle propostas pelo Ministério da Saúde tornem-se mais eficazes, e também que profissionais de saúde sejam capacitados para atuarem nos serviços rotineiramente.

\section{Referências}

1. Basano, S.A.; Camargo, L.M.A. Leishmaniose tegumentar americana: histórico, epidemiologia e perspectivas de controle. Rev. Bras. Epidemiol: 2004;7(3):328-77.

2. Braga, P.E.T.; Figueiredo, M.F.; Oliveira, D.A.S. Perfil epidemiológico dos casos de leishmaniose tegumentar americana na Serra da Meruoca, Ceará, no período de 2001 a 2012. SANARE: 2014;13(2):36-41.

3. Brasil. Ministério da Saúde. Secretaria de Vigilância em Saúde. Manual de vigilância da leishmaniose tegumentar americana. $2^{\mathrm{a}} \mathrm{ed}$. Brasília - DF, 2007.

4. OMS. Série de informes técnicos; 949: Control de las leishmaniasis: informe de uma reunión del Comité de Expertos de la OMS sobre el Control de las Leishmaniasis. Ginebra, 22-26/03/2010.
5. Brasil, 2016. Ministério da Saúde. Sistema de Informação de Agravos de Notificação. Disponível em $<$ http://www2.datasus.gov.br/DATASUS/inde x.php?area $=0203 \& i d=29878153>$. Acessado em: 25/05/2018. Brasil (b). Ministério da Saúde. Guia de Vigilância em Saúde. Brasília - DF; 2016.

6. Barbosa, R.N. Leishmaniose tegumentar em AIDS: manifestações clínicas e evolução. Dissertação (Mestrado em Medicina) - USP. São Paulo, 2006.

7. Lewis, D.J.; Ward, R.D. Transmission and vectors. In: Peter, W.; Killick-Kendrick, R. editores. The leishmaniases in biology and medicine. London: Academic Press: 1987;1:235-62.

8. Laison R, Shaw J.J, Silveira FT. Dermal and visceral leismaniasis and their causative agentes. Trans $\mathrm{R}$ Soc Med Hyg: 1987;81(4):702-3.

9. Brasil. Ministério da Saúde. Leishmaniose Tegumentar Americana: descrição da doença. Última atualização: 02/07/2014. Disponível em: $<$ http://portalms.saude.gov.br/saude-de-az/leishmaniose-tegumentar-americanaIta/11324-descricao-da-doenca> . Acessado em: 01/07/2018.

10. Carvalho M.L, Gontilho B. American cutaneous leishmaniasis. Rev Soc Bras Med Trop. 2003;36(1):71-80.

11. Falqueto $A$. et al. Epidemiological and clinical features of Leishmania (Viannia) braziliensis. American cutaneous and mucocutaneaous leishmaniasis in the Steta of Espirito Santo, Brazil. Mem Inst Oswaldo Cruz. 2003;98(8):1003-10.

12. Falqueto A, Sessa P.A. Leismaniose tegumentar americana. In: Veronesi, R.; Focaccia, R. editores. Tratado de Infectologia. 3ed. São Paulo: Atheneu; 2005;2:1543-1558.

13. Puig L, Pradinaud R. Leishmania and HIV com-infection: dermatological manifestations. Ann Trop Med Parasitol. 2003;97(1):107-14.

14. Coelho-Neto G.T. et al. Estudo epidemiológico de pacientes com leishmaniose tegumentar americana em Buriticupu, Pré-Amazônia maranhense. Rev. Ciênc. Saúde. 2012;14(2):133-138.

15. IBAMA. Instituto do Meio Ambiente e dos Recursos Naturais Renováveis. Principais resultados quantitativos do IBAMA no Maranhão anos 2009 e 2010. São Luís: 
Superintendência do IBAMA no Maranhão. MIMEO; 2011.

16. Muniz F.H. Efeito do manejo florestal sobre a composição florística e fitossociologia da floresta na Amazônia maranhense. In: Martins MB e Oliveira TG (Org.). Amazônia Maranhense: Diversidade e Conservação. Belém: MPEG; 2011.

17. Brasil. Ministério da Saúde. Conselho Nacional de Saúde. Resolução № 466 de 12 de dezembro de 2012. Diretrizes e normas regulamentadoras de pesquisas envolvendo seres humanos. Diário Oficial da União da República Federativa do Brasil, junho de 2013.

18. Vasconcelos P.P, Araujo N.J, Rocha F.J.S. Ocorrência e comportamento sociodemográfico de pacientes com leishmaniose tegumentar americana em Vicência, Pernambuco, no período de 2007 a 2014. Semina: Ciências Biológicas e da Saúde. 2017:38(1):105-114.

19. Oliart-Guzman H.; et al. Características epidemiológicas da leishmaniose tegumentar americana na fronteira Amazônica: estudo retrospectivo em Assis Brasil, Acre. Rev de Pat Trop. 2013;42(2):187-200.

20. Goes M.A.O.; Melo C.M.; Jeraldo V.L.S. Série temporal da leishmaniose visceral em Aracaju, estado de Sergipe, Brasil (1999 a 2008): aspectos humanos e caninos. Rev Bras Epidemiol. 2012:15(2):298-307.

21. Silva, N.S.; Muniz, V.D. Epidemiologia da leishmaniose tegumentar americana no Estado do Acre, Amazônia Brasileira. Cad Saúde Pública. v.25, n.6, p.1325-36. 2009.

22. Feliz G.C, et al. Perfil epidemiológico de pacientes com leishmaniose tegumentar americana no município de Barbalha-CE. Rev de Psicologia. 2011;5(14):30-5.

23. IBGE. Instituto Brasileiro de Geografia e Estatística. Censo Demográfico; 2010.

24. Oliveira R.Z., et al. Leishmaniose tegumentar americana no município de Jussara, estado do Paraná, Brasil: série histórica de 21 anos. Rev Saúde Pública Paraná. 2016:17(2):5965.

25. Gontijo C.M.F, Melo M.N. Leishmaniose visceral no Brasil, quadro atual, desafios e perspectivas. Rev Bras Epidemiol. 2004;7(3):338-9.

26. Murback N.D.N.; et al. Leishmaniose tegumentar americana: estudo clínico, epidemiológico e laboratorial realizado no Hospital Universitário de Campo Grande, Mato Grosso do Sul, Brasil. An Bras Dermatol. 2011;86(1):55-63.

27. Pelissari D.M, et al. Tratamento da leishmaniose visceral e leishmaniose tegumentar americana no Brasil. Rev. Epidemiol. Serv. Saúde. 2011;20(1):107-10.

28. Pontello Jr.R, Gon A.S, Ogama A. American cutaneous leishmaniasis: epidemiological profile of patients treated in Londrina from 1998 to 2009. An. Bras. Dermatol. 2013:88(5):748-53. 